\title{
An Uncommon Presentation of Cysticercosis Diagnosed on Fine Needle Aspiration Cytology with Brief Review of Literature
}

\author{
Manjari Kishore and Prajwala Gupta* \\ Dept of Pathology, PGIMER, Dr RML Hospital, New Delhi (India)
}

\begin{abstract}
Cysticercosis in human is caused by infection with the larval stage of Taenia solium. Oro-facial involvement of cysticercosis is not commonly reported. We present an uncommon case of subcutaneous cysticercosis diagnosed on fine needle aspiration cytology presenting as a solitary facial nodule in a young male.
\end{abstract}

Keywords: Cysticercosis, FNAC, Subcutaneous, Taenia Solium

\section{Introduction}

Cysticercosis is a parasitic infection caused by larvae of Taenia solium; commonly called as pork tapeworm and is usually seen reported in Southern Africa, India, Southeast Asia and Europe. The encysted larvae can be seen in almost any tissue. ${ }^{[1,2]}$ The commonly involved sites are central nervous system, subcutaneous tissue, striated muscle, vitreous humour of eye and rarely, other tissues. ${ }^{[1,2]}$ However, few cases have been reported in oro-facial region and poses difficulty in clinical diagnosis. ${ }^{[3,4]}$ Here, we report a case of subcutaneous cysticercosis over the maxillary region of face which was diagnosed on cytology.

\section{Case Report}

A 27-year-old male presented to surgery out-patientdepartment with asymptomatic subcutaneous nodule over left zygomatic process for last 2 years with progressive increase in size. On examination, the subcutaneous swelling measured $1 \mathrm{X} 1 \mathrm{~cm}$ and was firm in consistency, non-fluctuant, mobile and non-tender [Fig 1A]. The overlying skin was unremarkable. Systemic examination of the patient was normal and there was no other swelling. Clinically, a provisional diagnosis of sebaceous cyst was given.

Complete blood count revealed mild eosinophilia (absolute eosinophil count: $600 / \mu \mathrm{L}$ ). Other routine hematological and biochemical parameters were within normal limit. Chest X-ray did not reveal any abnormality. Ultrasound of the maxillary region showed a small oval hypoechoic lesion measuring $13.4 \mathrm{X} 7.6 \mathrm{~mm}$ with internal echoes and fine septa over left maxillary area in subcutaneous region suggestive of sebaceous cyst or parasitic infestation [Fig
1B]. The adjacent muscle and soft tissue did not show any abnormality.

Fine needle aspiration was done and smears prepared were air dried and fixed in 95\% ethyl alcohol and subsequently stained with Giemsa and Papanicolaou stain, respectively. Smears examined showed fragments of fibrillary material with interspersed small nuclei, suggestive of wall of bladder wall of parasite, i.e. cysticercus [Figure 2A, Giemsa \& 2B, Papanicolaou]. The background showed mixed inflammatory cells consisting of neutrophils, eosinophils, lymphocytes along with proteinaceous background. A final diagnosis of subcutaneous cysticercosis was made. After direct questioning, the patient revealed history of intake of pork since childhood.

The patient was advised to stop intake of pork and started on albendazole $400 \mathrm{mg}$ once daily for 21 days. After completion of antihelminthic therapy, the swelling significantly decreased in size. Further, Non-contrast computed tomography (NCCT) was done and did not show any evidence of neurocysticercosis or any neurological involvement. The patient is currently doing well.

\section{Discussion}

Taenia solium or the pork tapeworm, the causative parasite of cysticercosis passes its life cycle in two hosts. ${ }^{[1,2]}$ Human is the definitive host where the adult worms live in the small intestine. The intermediate host is pig which harbours the larval stage and leads to the causation of cysticercosis. ${ }^{[2,3]}$

Adult worm measures 3 meters in length with 1000 proglottids. Each gravid segment has about 50,000 eggs. The adult worm sheds these gravid segments along with eggs in stool which are taken up by pigs, which act as 


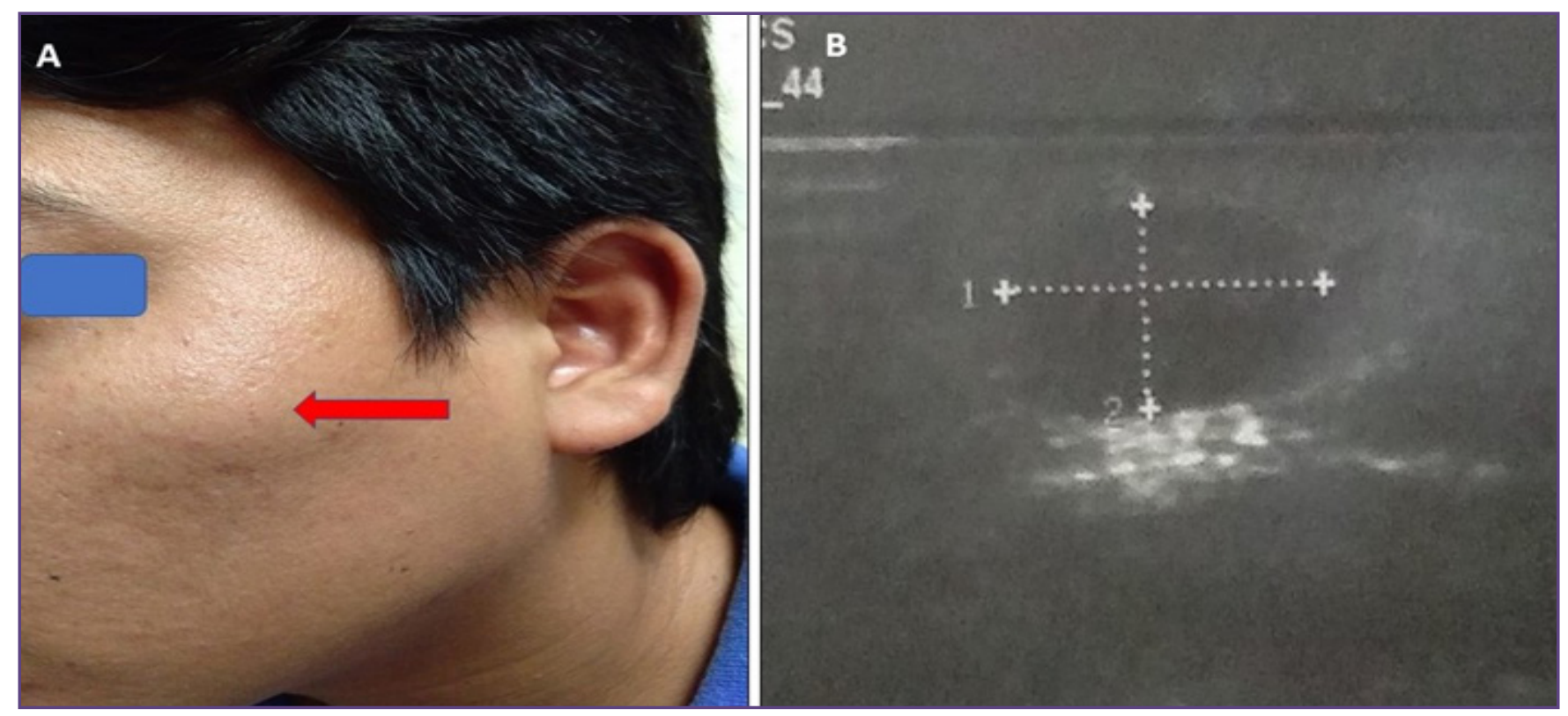

Fig. 1: 1A- A firm, mobile, non-fluctuant and non-tender subcutaneous swelling noted on upper cheek. 1B- Ultrasound of maxillary region showing a small oval hypoechoic lesion $(13.4 \times 7.6 \mathrm{~mm})$ with internal echoes \& fine septa in subcutaneous region suggestive of sebaceous cyst or parasitic infestation..

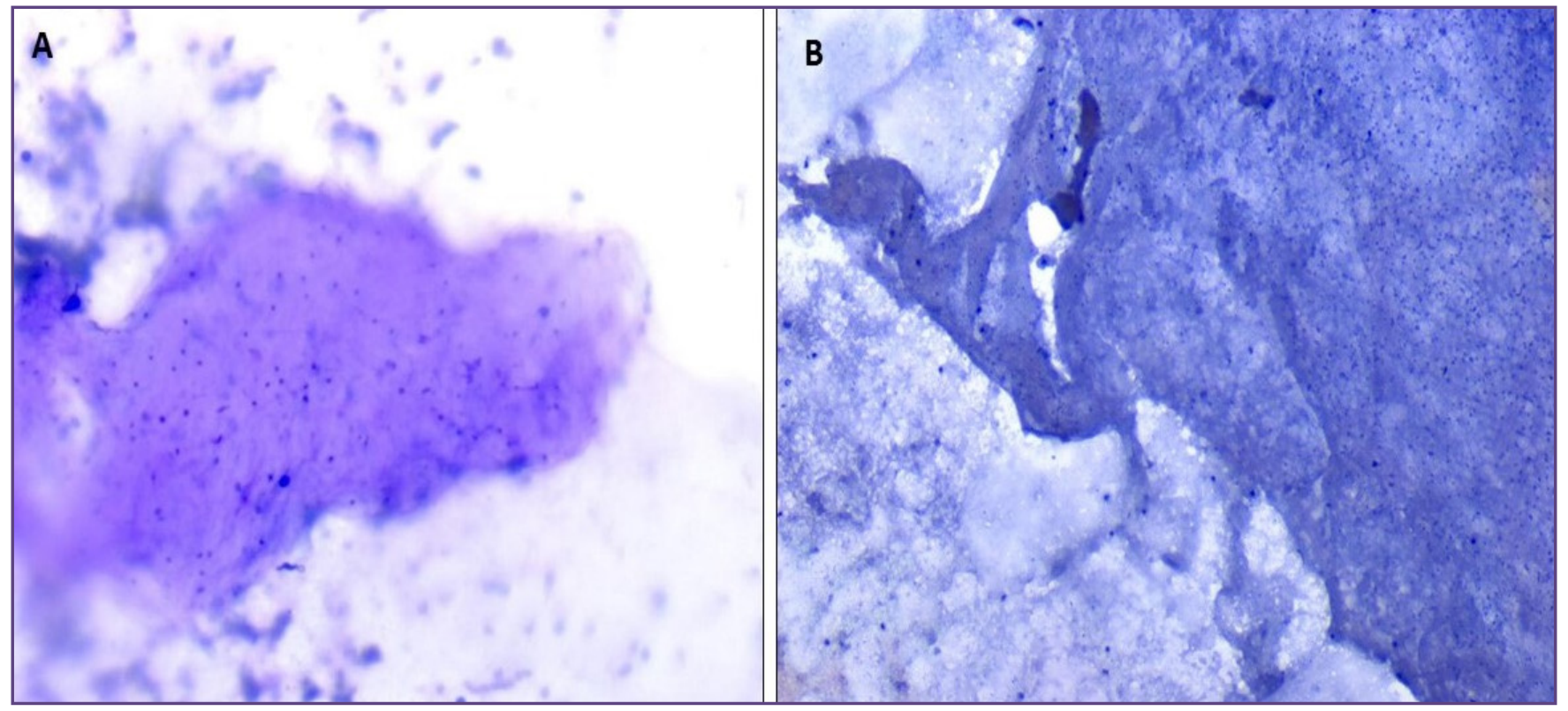

Fig. 2: A\&B- Cytological smears showing fragments of fibrillary material with interspersed small nuclei, suggestive of wall of bladder wall of parasite, i.e. cysticercus [2A, Giemsa- 200X and 2B, Papanicolaou-100X, resepectively].

the intermediate host. In the alimentary canal of the intermediate host, these eggs rupture and oncospheres are liberated. These oncospheres penetrate the gut wall of pigs and reach the systemic circulation and finally get lodged in various organs and muscles. It is over here that encystment of larval forms takes place and is termed as cysticercosis cellulosae. These larval forms can enter humans either by ingestion of contaminated food or water. The transmission of eggs via hands or carriers of the adult worm and by internal autoinfection by regurgitation of eggs into the stomach may also lead to infection. ${ }^{[4-6]}$

These larval forms reach the subcutaneous tissue, striated muscle, brain and ocular tissues via arteriovenous channels and lymphatics. Clinically, the patient may remain 
asymptomatic or present with epigastric discomfort, nausea and diarrhoea. Sometimes, these larval encystment occur in oral and facial region; however, these are rare. In majority of cases, cysticercosis presents with a solitary nodule and can be noted on trunk, upper arm, eyes, neck and rarely tongue, face and breast. In neurocysticercosis, the patient present with seizures and can be associated with skin nodules.

Radiological findings are not routinely used in the diagnosis of cysticercosis. ${ }^{[7]}$ These are cysticercus cyst with an inflammatory mass around it, irregular cyst with minimal fluid on one side. These may appear as a large irregular collection of exudative fluid or seen as calcified mass. ${ }^{[7]}$

The clinical differential diagnosis of facial cysticercosis can be varying like fibroma, lipoma, mucocele, pyogenic granuloma, sebaceous cyst or pleomorphic adenoma. ${ }^{[-7]}$ FNAC is a single tool helping in confirming the diagnosis of cysticercosis. However, the patient should be properly screened for any ocular and neurological involvement. The presence of fragment of larval bladder wall, hooklets and calcareous corpuscles confirms the diagnosis of cysticercosis. The background may be granular and reveal eosinophils, neutrophils, palisading histiocytes, giant cells. ${ }^{[7-9]}$ Cytologically, other differential diagnosis which can be kept in mind are hydatid cyst which shows presence of protoscolices, hooklets, and fragments of the laminated membrane. In some cases, only laminated membranes are present in the inflammatory background. Cysticercosis must also be differentiated from abscesses and nonparasitic cysts. ${ }^{[10]}$

The standard treatment for facial cysticercosis is institution of antihelminthic drugs like praziquantel and albendazole. ${ }^{[7-9]}$ Sometimes, corticosteroids are used in cases where there is associated inflammatory reaction. Effective preventive measures can also be taken like consumption of well-washed vegetables, filtered or boiled water and proper hand washing before meals and preparation of food.

\section{Conclusion}

Although, lesions in the facial region are uncommon, cysticercosis should be kept as a clinical differential diagnosis in conjunction with dietary history and ultrasound findings. FNAC is a simple and quick mode of investigation in diagnosing clinically unsuspected case of cysticercosis of facial region, so that early antihelminthic therapy can be started. However, the patient should be followed for any ophthalmologic or neurologic signs of cysticercosis cellulosae to prevent any complications.

\section{References}

1. Sidhu R, Nada R, Palta A, Mohan H, Suri S., et al. Maxillofacial cysticercosis: Uncommon Appearances of a Common Disease. J Ultrasound Med 2002;21:199-202.

2. Pandey S.C., Pandey S.D. Lingual cysticercosis. Indian J Plast Surg 2005;38:160

3. Reddi SP, Molares MJ, Addante RD. Solitary lesion in the masseter muscle. J Oral Maxillofac Surg 2001;59:71-5.

4. Goyal P, Ghosh S, Sehgal S, Mittal D, Singh S. Solitary Cysticercosis of Parotid Gland Diagnosed on FNAC. APSP Journal of Case Reports. 2014;5(1):11.

5. Bandyopadhyay D, Sen S. Disseminated cysticercosis with huge muscle hypertrophy: Indian J Dermatol 2009;54:49-51.

6. Mittal A, Das D, Iyer N. Masseter cysticercosis - a rare case diagnosed on ultrasound. Dentatomaxillofac Radiol 2008;37:113-16.

7. Kala P, Khare P. Fine-needle aspiration cytology as a diagnostic modality for cysticercosis: A clinicopathological study of 137 case.J Cytol.2014;31:68-72.

8. Chaurasia RN, Garg RK, Agarwall A. Three day albendazole therapy in patients with a solitary cysticercus granuloma: a randomized double blind placebo controlled study. Southeast Asian J Trop Med Public Health 2010;41:517-25.

9. Shetty RM, Ramprasad, Kalra A, Goyal S, Sachin BM., et al. Cysticercosis cellulosae in labial mucosa: a rare case report. J Indian Acad Oral Med Radio 2010; 22: 548-50.

10. Kim AR, Park SJ, Gu MJ, Choi JH, Kim HJ et al. Fine Needle Aspiration Cytology of Hepatic Hydatid Cyst: A Case Study. Korean J Pathol. 2013; 4: 395-398.

*Corresponding author:

Dr Prajwala Gupta, Room No.302, 3rd floor, OPD building, Dr RML Hospital, Baba Khadak Singh Maarg, New Delhi(India)-110001

Phone: +91 991036227

Email: prajwala2000@yahoo.com

Financial or other Competing Interests: None.

Date of Submission : 28.07.2017

Date of Acceptance : 17.09.2017

Date of Publication : 13.01.2018 